\title{
Bilateral Suprascapular Nerve Entrapment by Ganglion Cyst Associated with Superior Labral Lesion
}

\author{
Giacomo Rizzello ${ }^{1,2}$, Umile Giuseppe Longo ${ }^{1,2}$, Ugo Trovato ${ }^{1,2}$, Caterina Fumo ${ }^{1,2}$, \\ Wasim Sardar Khan ${ }^{*, 3}$, Nicola Maffulli ${ }^{4}$ and Vincenzo Denaro ${ }^{1,2}$
}

\author{
${ }^{I}$ Department of Orthopaedic and Trauma Surgery. Campus Bio-Medico University, Via Alvaro del Portillo, 200, 00128 \\ Trigoria, Rome, Italy. \\ ${ }^{2}$ Centro Integrato di Ricerca (CIR) Campus Bio-Medico University, Via Alvaro del Portillo, 21, 00128, Rome, Italy \\ ${ }^{3}$ University College London Institute of Orthopaedics and Musculoskeletal Sciences, Royal National Orthopaedic \\ Hospital, Stanmore, HA7 4LP, UK \\ ${ }^{4}$ Centre for Sports and Exercise Medicine, Barts and The London School of Medicine and Dentistry, Mile End Hospital, \\ 275 Bancroft Road, London E1 4DG, UK
}

\begin{abstract}
Suprascapular nerve compression is a rare cause of shoulder pain. We report the clinical features, radiological findings, arthroscopic management and outcome of three patients with suprascapular nerve compression caused by labral ganglion cyst associated with SLAP lesion. We performed simultaneous suture anchor SLAP repair and cyst decompression with a blunt probe. Upon a two-year follow-up, patients recovered full shoulder function without pain or limitations in activities of daily living.
\end{abstract}

Keywords: Arthroscopy, suprascapular nerve compression, ganglion cyst, SLAP, shoulder.

\section{INTRODUCTION}

Suprascapular nerve entrapment can be a consequence of direct trauma [1-3], anterior dislocation or fractures of the scapula [4] and overhead sports activities [3, 5]. Direct compression of the nerve can occur by progressive compressive masses of suprascapular notch or spinoglenoid notch as a consequence of malignant tumors [1, 6-8], haematomas or ganglion cysts [1,2, 9-15].

Clinically, suprascapular nerve entrapment is characterized by deep, diffuse posterolateral shoulder pain, which may radiate to the neck, arm, or upper chest wall. Entrapment syndrome is diagnosed on the basis of the clinical history, physical examination and electromyography $[10,16,17]$.

We report the clinical features, radiological findings, arthroscopic management and outcome of two patients with suprascapular nerve compression caused by labral ganglion cyst associated with SLAP lesion. In both patients arthroscopy allowed decompression of the labral ganglion cyst and repair of the SLAP lesion.

\section{CASE REPORTS}

\section{Patient 1}

A 35-year old cleaning woman presented with a one year history of bilateral shoulder pain and weakness in the

*Address correspondence to this author at the Clinical Lecturer, University College London Institute of Orthopaedics and Musculoskeletal Science, Royal National Orthopaedic Hospital, Stanmore, London, HA7 4LP, UK; Tel: +44 (0) 7791 025554; Fax: +44 (0) 208570 3864;

E-mail: wasimkhan@doctors.org.uk scapular girdle. She was managed conservatively for both shoulder with nonsteroidal anti-inflammatory drugs, physiotherapy, rest and local injections of corticosteroids with no benefits. Clinical examination showed tenderness and pain in the suprascapular and subscapular spaces and subspinosus hypotrophy in both shoulders. O'Brien test and Speed test were positive. Plain radiographs were unremarkable. Magnetic resonance imaging scans of the right shoulder revealed a $4 \times 2.5 \mathrm{~cm}$ mass with low signal intensity on T1-weighted sequences and high signal intensity on T2-weighted sequences, just below the supraspinatus muscle. Magnetic resonance imaging scans of the left shoulder revealed a $3 \times 1.5 \mathrm{~cm}$ well-defined mass involving the spinoglenoid notch. Bilateral electromyography was performed showing signs of bilateral suprascapular nerve entrapment neuropathy. Arthroscopy of her right shoulder was performed showing a type II SLAP lesion associated with a cyst into the spinoglenoid notch. A blunt probe was used for dissection to the spinoglenoid notch. A digital pressure over the infraspinatus fossa was associated to decompress the cyst into the joint through the labral defect. Labral repair was then performed. Postoperatively the arm was hold in a sling with an abduction pillow for 4 weeks. Passive exercises were allowed after the $2^{\circ}$ week and active exercises were allowed after the $3^{\circ}$ week. Six months later the patient underwent arthroscopy of her left shoulder. Intraoperative findings showed a type I SLAP lesion and a ganglion cyst in the spinoglenoid notch. The same surgical management was performed. MRI scans of both shoulders performed 1 year after the first arthroscopy showed that the cystic masses had dissolved and electromyographic findings showed suprascapular reinnervation signs. 


\section{Patients 2}

A 57-year-old housewife presented with a 9 months history of pain and weakness in the right shoulder. The patient was managed for 2 months with nonsteroidal antiinflammatory drugs, physiotherapy, rest and one local injection of corticosteroid. On examination, the patient had tenderness and pain in the suprascapular and subscapular spaces and subspinosus hypotrophy. The patient had a full range of motion, but lack of strength in external rotation of abducted arm. O'Brien test and Speed test were positive. Plain radiographs were unremarkable. Magnetic resonance imaging scans of the shoulder revealed a 5 x $3 \mathrm{~cm}$ welldefined mass involving the spino-glenoid notch. The mass had low signal intensity on T1 MRI images and high signal intensity on T2MRI images (Fig. 1I, II). Electromyography showed increased latency time of suprascapular nerve and one run short of positive sharp waves in the supraspinatus muscle. These findings were consistent with a suprascapular nerve entrapment neuropathy. The patient underwent arthroscopy of her right shoulder, which revealed a type III SLAP lesion (Fig. 2). A blunt probe was used for dissection to the spinoglenoid notch. A digital pressure over the infraspinatus fossa was associated to decompress the cyst into the joint through the labral defect (Fig. 3). A repair of the SLAP lesion was then performed with suture anchors. Postoperatively the arm was hold in a sling with an abduction pillow for 4 weeks. Passive exercises were allowed after the $2^{\circ}$ week and active exercises were allowed after the $3^{\circ}$ week. Two months after arthroscopic cyst decompression, an MRI showed that the cystic mass had dissolved; electromyographic findings showed suprascapular reinnervation signs.

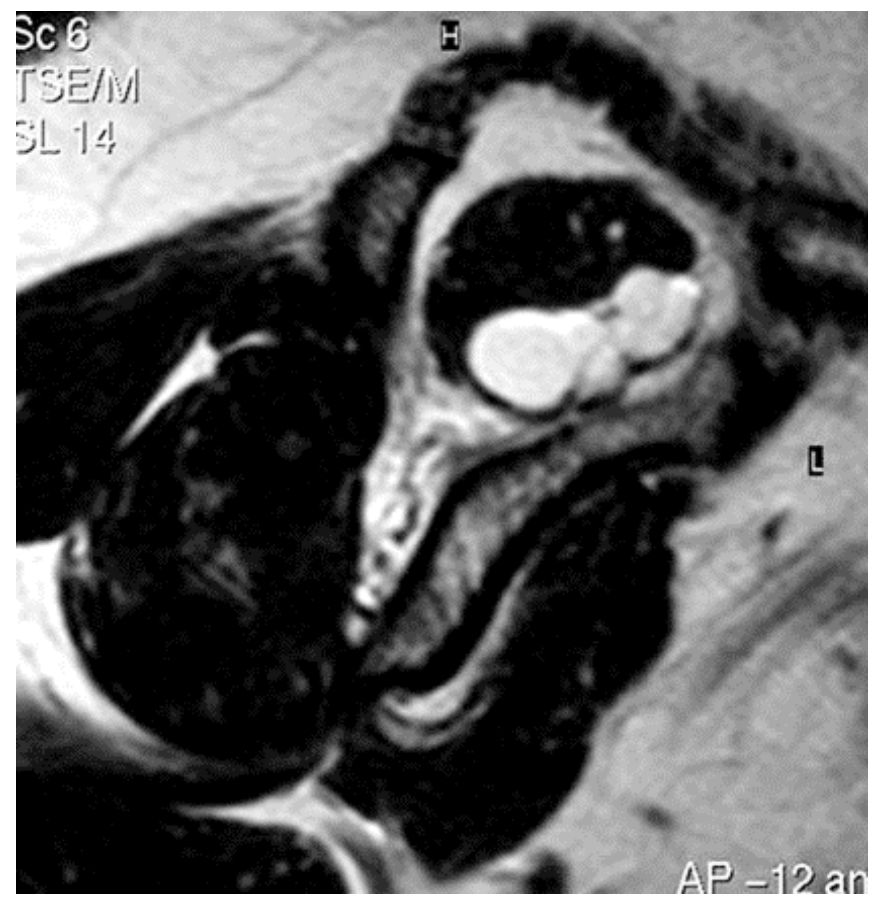

Fig. (1). Preoperative T2 weighted sagittal MRI of a shoulder showing cyst formation at spinoglenoid notch.

\section{Patient 3}

A 28-year-old housewife presented with one year history of pain and weakness in her left shoulder. 3 months of conservative management had previously failed. On clinical examination, the patient showed subspinosus hypotrophy and lack of strength in external rotation. Electromyography showed suprascapular nerve entrapment neuropathy signs: increased latency time of suprascapular nerve. Magnetic resonance imaging scans of the shoulder revealed a $4 \times 2 \mathrm{~cm}$ well-defined mass just below the supraspinatus muscle. Superior labrum showed abnormal signal intensity suggesting a structural continuity with ganglion cyst (Fig. 4I, II). The patient underwent arthroscopy of her left shoulder, which revealed a type II SLAP lesion. Dissection with a blunt probe as previously described and labral repair were performed (Fig. 5). Two months after arthroscopic cyst decompression, an MRI showed that the cystic mass had dissolved (Fig. 6) and electromyographic findings showed suprascapular reinnervation signs.

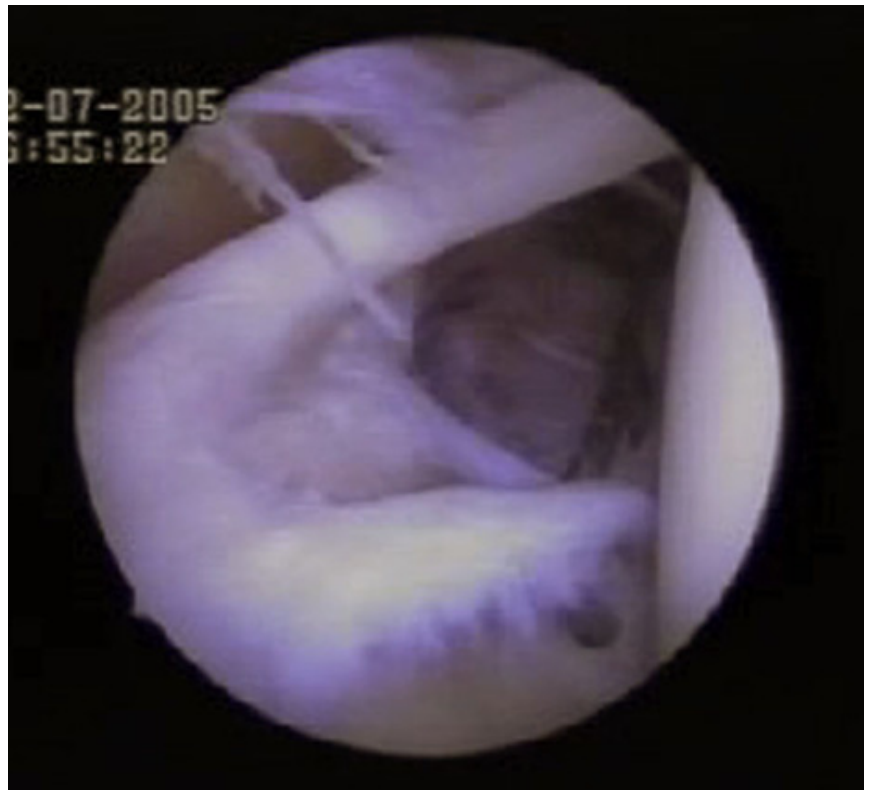

Fig. (2). Arthroscopic view from a posterior portal showing a type III SLAP lesion.

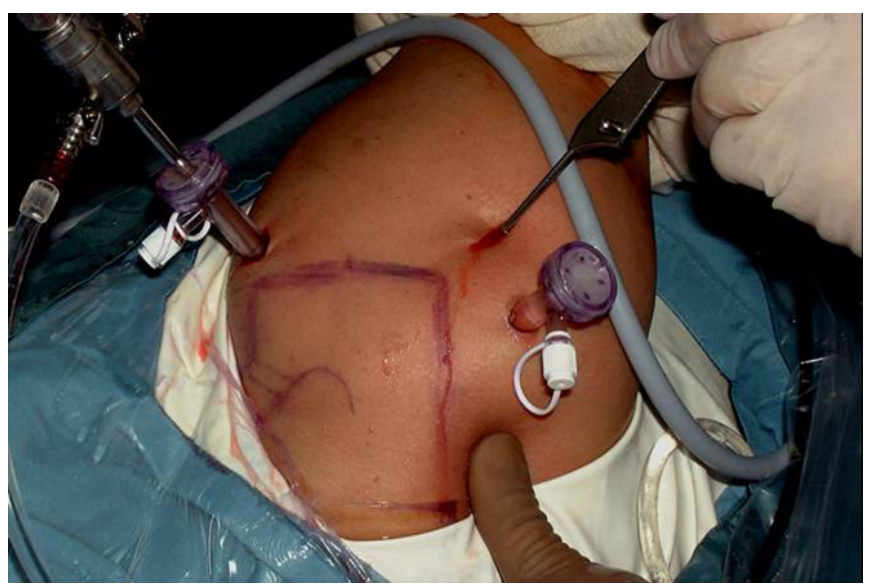

Fig. (3). The probe is inserted through a postero-lateral portal and digital pressure is applied over the infraspinatus fossa.

\section{DISCUSSION}

Suprascapular nerve is a branch of the superior trunk of the brachial plexus. It runs laterally beneath the trapezius parallel to the omohyoid muscle, entering the supraspinous 
fossa through the suprascapular notch.. There are many variations in the anatomy of the suprascapular notch: 6 anatomic variants of the suprascapular notch are described in literature $[12,13]$, that can be considered ad potential etiologic factors for entrapment of the suprascapular nerve $[18,19]$. The suprascapular nerve has both a motor component (innervating the infraspinatus and supraspinatus muscles) and a sensory component (supplying the glenohumeral and acromioclavicular joints).

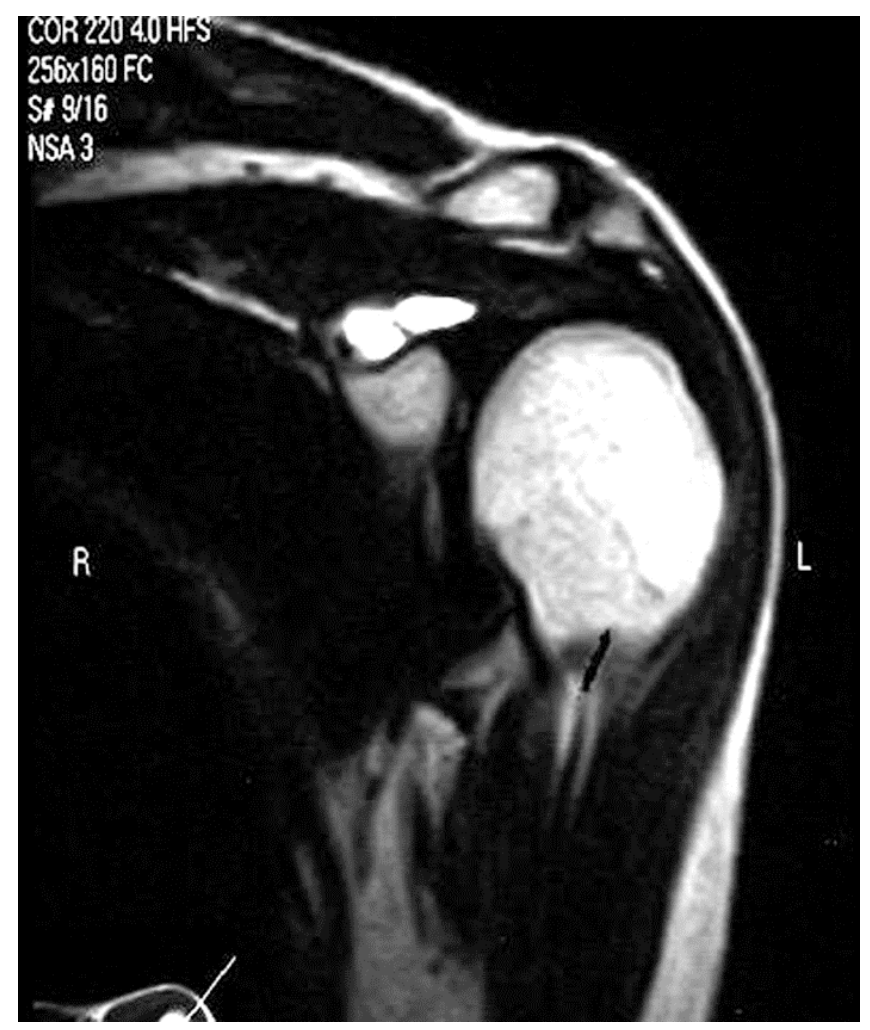

Fig. (4). Preoperative T2 weighted coronal MRI of a shoulder showing cyst formation at spinoglenoid notch.

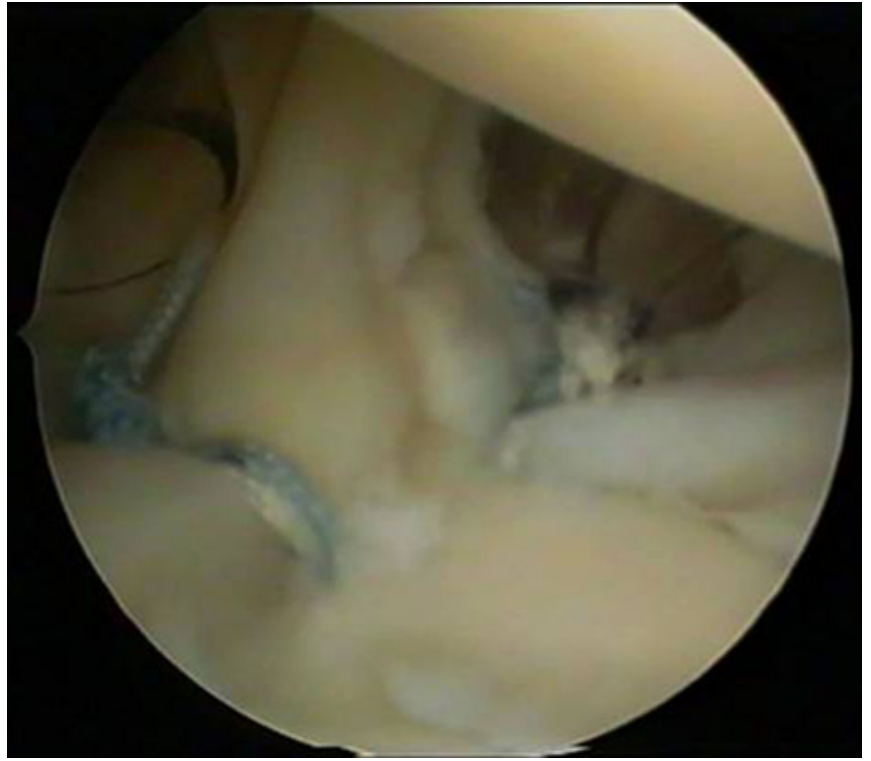

Fig. (5). Arthroscopic view from a posterior portal showing a repaired SLAP II lesion.

Ganglion cyst is an unusual cause of entrapment of the suprascapular nerve [9].

Even though the pathogenesis of labral ganglion cysts remains unclear, the most widely held theory is that labral ganglion cysts simply result from sinovial fluid extrusion through labral tears $[14,16,17]$. The diagnosis of the entrapment syndrome is based on the history, physical findings and the abnormal electromyographic findings of the affected muscles [10, 16, 17]. The differential diagnosis should include local diseases and systemic diseases [10, 16, $17,20]$ such as glenohumeral instability, cervical disc disease, cervical spondylosis, brachial plexopathy, rotator cuff or labral pathology, glenohumeral and acromioclavear osteoarthritis, peripheral neuritis or neuropathies, Pancoast disease and thoracic out-let disease.

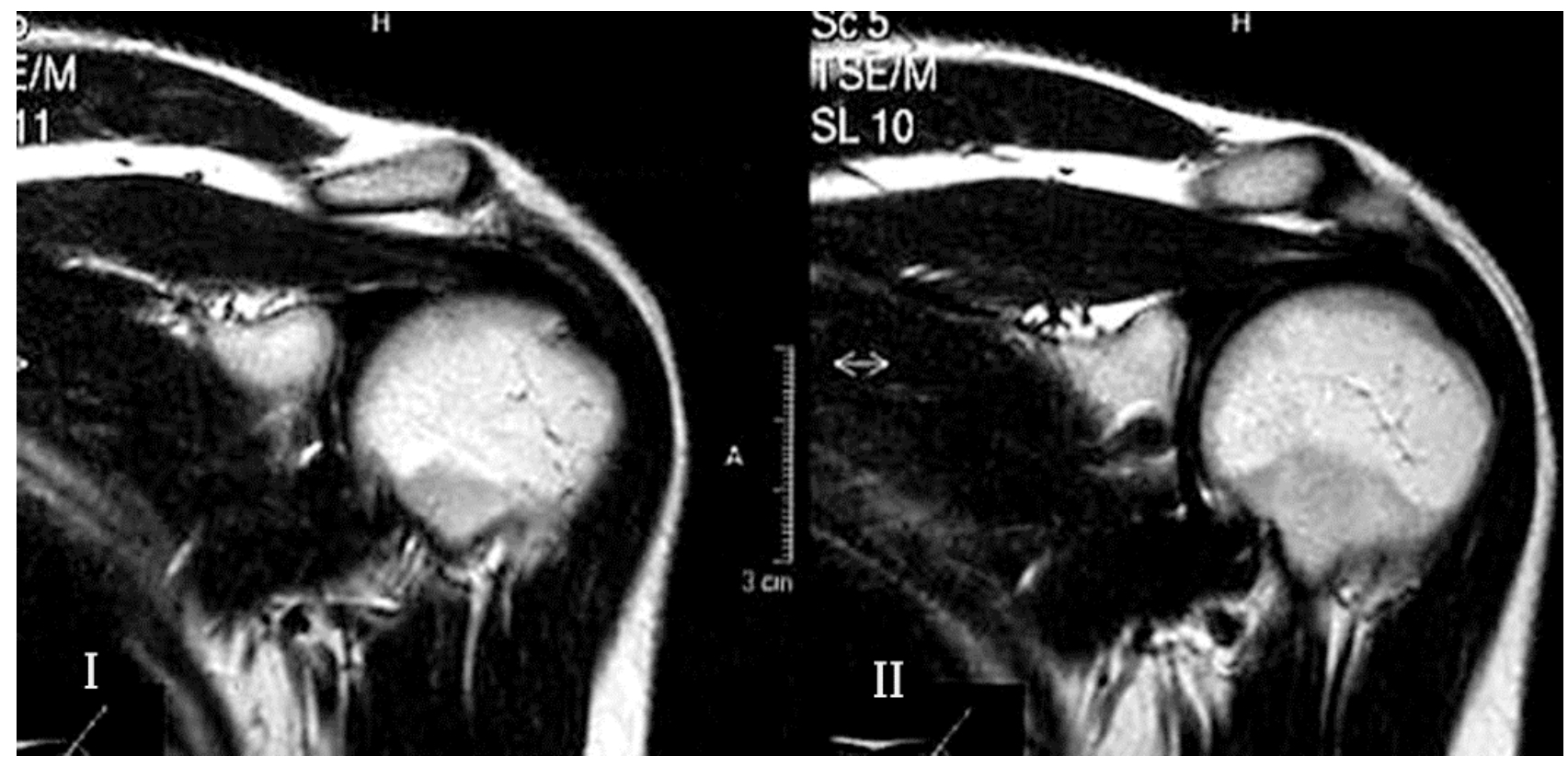

Fig. (6). (I, II) Postoperative MRI control of the same patient showing no recurrence of the cyst formation. 
MRI are routinely used to investigate labral ganglion cysts, and have the advantage of avoiding radiation $[1,15$, 21]. Ganglion cyst appears as a well defined, smoothly mass. MRI is helpful for diagnosis of associated lesions such as muscle denervation, showed by an high intensity signal, and evaluating muscle recovery after nerve decompression [21].

Chochole et al. [22] reported on arthroscopic repair of the SLAP lesion without drainage of the cyst in one case showing total resolution of the cyst documented on MRI.

In our patients we performed simultaneous suture anchor SLAP repair and cyst decompression with a blunt probe, through the labral defect associated with digital pressure over the infraspinatus fossa [23].

Repair of labral lesion is a necessary step in the management of suprascapular nerve entrapment by ganglion cyst associated with superior labral lesion. Since labral cysts are probably a consequence of drainage of joint fluid out of the joint through labral lesions [2, 11, 14, 15, 18, 22, 24-28], labral repair is important in avoiding cyst recurrence.

\section{CONCLUSIONS}

In conclusion, arthroscopy is a safe procedure to manage ganglion cyst associated with superior labral lesion causing suprascapular nerve compression. Further studies are necessary to evaluate the etiopathogenesis of these conditions.

\section{CONFLICT OF INTEREST}

The authors confirm that this article content has no conflict of interest.

\section{ACKNOWLEDGEMENTS}

Declared none.

\section{REFERENCES}

[1] Fritz RC, Helms CA, Steinbach LS, Genant HK. Suprascapular nerve entrapment: evaluation with MR imaging. Radiology 1992; 182: $437-44$

[2] Ferrick MR, Marzo JM. Suprascapular entrapment neuropathy and ganglion cysts about the shoulder. Orthopedics 1999; 22: 430-4; discussion 434-5; quiz 436-7.

[3] Aiello I, Serra G, Traina GC, Tugnoli V. Entrapment of the suprascapular nerve at the spinoglenoid notch. Ann Neurol 1982; 12: 314-6.

[4] Solheim LF, Roaas A. Compression of the suprascapular nerve after fracture of the scapular notch. Acta Orthop Scand 1978; 49: $338-40$.

[5] Thompson WA, Kopell HP. Peripheral entrapment neuropathies of the upper extremity. N Engl J Med 1959; 260: 1261-5.

[6] Gelberman RH, Verdeck WN, Brodhead WT. Supraclavicular nerve-entrapment syndrome. J Bone Joint Surg Am 1975; 57: 119.

[7] Hazrati Y, Miller S, Moore S, Hausman M, Flatow E. Suprascapular nerve entrapment secondary to a lipoma. Clin Orthop Relat Res 2003: 124-8.
[8] Zvijac JE, Sheldon DA, Schurhoff MR. Extensive lipoma causing suprascapular nerve entrapment. Am J Orthop 2003; 32: 141-3.

[9] Antoniadis G, Richter HP, Rath S, Braun V, Moese G. Suprascapular nerve entrapment: experience with 28 cases. J Neurosurg 1996; 85: 1020-5.

[10] Ganzhorn RW, Hocker JT, Horowitz M, Switzer HE. Suprascapular-nerve entrapment. J Bone Joint Surg Am 1981; 63: 492-4.

[11] Ferrick MR, Marzo JM. Ganglion cyst of the shoulder associated with a glenoid labral tear and symptomatic glenohumeral instability. A case report. Am J Sports Med 1997; 25: 717-9.

[12] Rengachary SS, Burr D, Lucas S, Hassanein KM, Mohn MP, Matzke H. Suprascapular entrapment neuropathy: a clinical, anatomical, and comparative study. Part 2: anatomical study. Neurosurgery 1979; 5: 447-51.

[13] Rengachary SS, Burr D, Lucas S, Brackett CE. Suprascapular entrapment neuropathy: a clinical, anatomical, and comparative study. Part 3: comparative study. Neurosurgery 1979; 5: 452-5.

[14] Moore TP, Fritts HM, Quick DC, Buss DD. Suprascapular nerve entrapment caused by supraglenoid cyst compression. J Shoulder Elbow Surg 1997; 6: 455-62.

[15] Tirman PF, Feller JF, Janzen DL, Peterfy CG, Bergman AG. Association of glenoid labral cysts with labral tears and glenohumeral instability: radiologic findings and clinical significance. Radiology 1994; 190: 653-8.

[16] Post M, Mayer J. Suprascapular nerve entrapment. Diagnosis and treatment. Clin Orthop Relat Res 1987: 126-36.

[17] Post M. Diagnosis and treatment of suprascapular nerve entrapment. Clin Orthop Relat Res 1999: 92-100.

[18] Urguden M, Ozdemir H, Donmez B, Bilbasar H, Oguz N. Is there any effect of suprascapular notch type in iatrogenic suprascapular nerve lesions? An anatomical study. Knee Surg Sports Traumatol Arthrosc 2004; 12: 241-5.

[19] Bayramoglu A, Demiryurek D, Tuccar E, et al. Variations in anatomy at the suprascapular notch possibly causing suprascapular nerve entrapment: an anatomical study. Knee Surg Sports Traumatol Arthrosc 2003; 11:393-8.

[20] Reid AC, Hazelton RA. Suprascapular nerve entrapment in the differential diagnosis of shoulder pain. Lancet 1979; 2: 477.

[21] Inokuchi W, Ogawa K, Horiuchi Y. Magnetic resonance imaging of suprascapular nerve palsy. J Shoulder Elbow Surg 1998; 7: 2237.

[22] Chochole MH, Senker W, Meznik C, Breitenseher MJ. Glenoidlabral cyst entrapping the suprascapular nerve: dissolution after arthroscopic debridement of an extended SLAP lesion. Arthroscopy 1997; 13: 753-5.

[23] Iannotti JP, Ramsey ML. Arthroscopic decompression of a ganglion cyst causing suprascapular nerve compression. Arthroscopy 1996; 12(6): 739-45.

[24] Chen AL, Ong BC, Rose DJ. Arthroscopic management of spinoglenoid cysts associated with SLAP lesions and suprascapular neuropathy. Arthroscopy 2003; 19: E15-21.

[25] Vastamaki M, Goransson H. Suprascapular nerve entrapment. Clin Orthop Relat Res 1993; 297: 135-43.

[26] Ozbaydar MU, Tonbul M, Baca E, Yalaman O. Arthroscopic treatment of anterior-inferior shoulder instability. Acta Orthop Traumatol Turc 2007; 41(2): 120-6.

[27] Ozcanli H, Aydin AT, Yeter AB, Akyidiz FF, Gürer EI. A new technique for aneurysmal bone cysts of the proximal humerus: cortical collapsing. Acta Orthop Traumatol Turc 2008; 42(3): 1615.

[28] Kiliç B, Uysal M, Cinar BM, Ozkoç G, Demirörs H, Akpinar S. Early results of treatment of proximal humerus fractures with the PHILOS locking plate. Acta Orthop Traumatol Turc 2008; 42(3): $149-53$. 